\title{
Erratum to: Quadratic mixed finite element approximations of the Monge-Ampère equation in 2D
}

\author{
Gerard Awanou ${ }^{1}$
}

Published online: 11 April 2016

(C) Springer-Verlag Italia 2016

\section{Erratum to: Calcolo (2015) 52:503-518 DOI 10.1007/s10092-014-0127-7}

\begin{abstract}
The proof of Lemma 10 in [Awanou, G.: Quadratic mixed finite element approximations of the Monge-Ampère equation in 2D. Calcolo 52(4), 503-518 (2015)] is not correct. The purpose of this erratum is to give a correct proof of the main result therein under the assumption of elliptic regularity.
\end{abstract}

\section{Introduction}

In [1, Lemma 10], we claimed a strict contraction property of a mapping $T_{1}$ in the $H^{1}$ seminorm. Unfortunately there was a mistake at the end of the proof of the lemma. It was stated that "Since $\gamma<1$, and $\alpha=h^{k+2}$, for $h$ sufficiently small, $C h+$ $C \alpha h\|\operatorname{cof} Q\|_{H^{k+1}\left(\mathcal{T}_{h}\right)}+C \alpha<1-\gamma$ ". However $\gamma$ also depends on $h$, see [1, p. 6]. Moreover $1-\gamma \rightarrow 0$ at a rate higher than $h$, and thus the argument as stated is not correct. As a consequence, the strategy which consists in rescaling the equation does not work.

In this erratum, using the same notation as in [1], we give a proof of the main result therein under the assumption of $W^{2, p}$ elliptic regularity. Our approach consists

The online version of the original article can be found under doi:10.1007/s10092-014-0127-7.

Gerard Awanou

awanou@uic.edu

http://www.math.uic.edu/ awanou

1 Department of Mathematics, Statistics, and Computer Science (M/C 249), University of Illinois at Chicago, Chicago, IL 60607-7045, USA 
in adapting the proof in [3]. The main ingredient is a $W^{2, p}$ discrete elliptic regularity proved in [13].

The elliptic regularity assumption is known to hold if the domain is smooth. We refer to [14, Remark 3.2] and [13] for the formulation of the method with the weak imposition of the Dirichlet boundary condition using Nitsche's method and the use of curvilinear coordinates near the boundary. The arguments given here can be extended to that setting.

On the other hand, $W^{2, p}$ elliptic regularity holds for the Poisson equation on a cube [17, Remark 9.1.1]. It is therefore reasonable to expect that one can prove a $W^{2, p}$ elliptic regularity result on cubes for second order equations in divergence form with smooth coefficients using an antisymmetric extension as in the proof of [17, Proposition 9.1.2]. We wish to address this issue, following the $W^{2, p}$ elliptic regularity approach in [11], in a separate work.

\section{Preliminaries}

We use the standard notation $W^{k, p}(\Omega)$ for the Sobolev spaces and the notation $|\cdot|_{W^{k, p}}$ for its semi norm. We recall that $W_{0}^{1, p}(\Omega)$ is the subset of $W^{1, p}(\Omega)$ of elements with vanishing trace on $\partial \Omega$. We will need the following mesh dependent norm on $V_{h}$

$$
\|v\|_{\widetilde{W}^{2, p}\left(\mathcal{T}_{h}\right)}^{p}=\|v\|_{W^{2, p}\left(\mathcal{T}_{h}\right)}^{p}+h^{1-p} \sum_{K \in \mathcal{T}_{h}}\|D v\|_{L^{p}(\partial K)}^{p}, \quad p \geq 2 .
$$

We have by scaling

$$
\begin{array}{ll}
\|v\|_{\widetilde{W}^{2, p}\left(\mathcal{T}_{h}\right)} \leq C\|v\|_{W^{2, p}\left(\mathcal{T}_{h}\right)}, \quad \forall v \in V_{h} \\
\|v\|_{\widetilde{W}^{2, p}\left(\mathcal{T}_{h}\right)} \leq C h^{-1}\|v\|_{W^{1, p}}, \quad \forall v \in V_{h} .
\end{array}
$$

Moreover, there exists an interpolation operator $\tilde{I}_{h}$ such that for $m \in W^{k+1, p}(\Omega) \cap$ $W_{0}^{1, p}(\Omega), \tilde{I}_{h} m \in V_{h} \cap W_{0}^{1, p}(\Omega)$ and

$$
\begin{aligned}
\left\|m-\tilde{I}_{h} m\right\|_{\widetilde{W}^{2, p}\left(\mathcal{T}_{h}\right)} & \leq C h^{k-1}|m|_{W^{k+1, p}} \\
\left\|m-\tilde{I}_{h} m\right\|_{W^{1, p}} & \leq C h^{k}|m|_{W^{k+1, p}} .
\end{aligned}
$$

The proofs are essentially the same as the ones given for [4, Lemma 1], [4, Lemma 2] and [4, Lemma 4]. It is important to note that the constant in the above inequalities are independent of $p$. This follows from the fact that the constant in the Bramble-Hilbert lemma [5, (4.3.9)] is independent of $p$.

We recall the scale-trace inequality

$$
\|v\|_{L^{p}(\partial K)} \leq C h^{-\frac{1}{p}}\|v\|_{L^{p}(K)} \leq C h^{-\frac{1}{2}}\|v\|_{L^{p}(K)}, \quad p \geq 2
$$

with a constant $C$ independent of $p$. 
We also recall that if $w$ is in the Sobolev space $W^{l+1, p}(\Omega), 1 \leq p \leq \infty, 0 \leq l \leq d$

$$
\| w-\left.I_{h} w\right|_{W^{k, p}\left(\mathcal{T}_{h}\right)} \leq C h^{l+1-k}|w|_{W^{l+1, p}},
$$

for $k=0,1,2$. The constant $C$ is shown to be independent of $p$ using [5, (4.4.5)] and shape regularity.

We will often use the inverse estimates

$$
\left\|w_{h}\right\|_{t, p, \mathcal{T}_{h}} \leq C h^{s-t+\min \left(0, \frac{n}{p}-\frac{n}{q}\right)}\left\|w_{h}\right\|_{s, q, \mathcal{T}_{h}}
$$

for $0 \leq s \leq t, 1 \leq p, q \leq \infty$ and $w_{h} \in V_{h}$. As stated in [5], the constant $C$ in (2.6) depends on $p$ and $q$ because the first step of the proof is to use a norm equivalence on the reference element. However, inspection of the proof of the equivalence of norms in a finite dimensional space reveals that the constant does not depend on $q$. Moreover, it only depends on $p$ through the $W^{t, p}$ norm of the basis functions of the finite dimensional space on the reference element. The latter are bounded by a scalar multiple of their $W^{t, \infty}$ norm. We conclude that the constant $C$ in (2.6) can be chosen independent of $p$.

Next, let $\phi$ be the solution of

$$
-\operatorname{div}\left(\left(\operatorname{cof} D^{2} u\right) D \phi\right)=r \text { in } \Omega, \quad \phi=0 \text { on } \partial \Omega \text {. }
$$

We make the following assumption

Assumption 2.1 For $r \in L^{p}(\Omega), p \geq 2$, the weak solution $\phi$ of (2.7) is in $W^{2, p}(\Omega)$ and

$$
\|\phi\|_{W^{2, p}} \leq C p\|r\|_{L^{p}} .
$$

The result is known to hold for smooth domains, c.f. [14] and the references therein. As suggested in [16, (1.7)] the linear dependence in $p$ of the constant in (2.8) follows by tracing constants in the proof given in [11]. Once can trace constants in the proof of [11, Theorem 9.14] and use the maximum principle [11, Theorem 9.1]. See also [7].

As pointed out in the introduction, it is reasonable to expect that the result also holds for cubes.

We will refer to the result of the following lemma as discrete elliptic regularity. The result is given as [13, Lemma 4.1]. For the convenience of the reader, we give the proof.

Let $P_{h}: W_{0}^{1, p}(\Omega) \rightarrow V_{h} \cap W_{0}^{1, p}(\Omega)$ be the projection defined by

$\int_{\Omega}\left[\left(\operatorname{cof} D^{2} u\right) D P_{h} v\right] \cdot D w d x=\int_{\Omega}\left[\left(\operatorname{cof} D^{2} u\right) D v\right] \cdot D w d x, \quad \forall w \in V_{h} \cap W_{0}^{1, p}(\Omega)$.

We have the approximation property

$$
\left\|w-P_{h} w\right\|_{W^{1, p}} \leq C h^{k}|w|_{W^{k+1, p}}
$$


The result is a consequence of the stability of the Ritz projection, [15] and [12, Corollary 5.6]. Since $\left\|P_{h} w\right\|_{W^{1, p}} \leq C\|w\|_{W^{1, p}}$ for $w \in W_{0}^{1, p}(\Omega)$, using $P_{h} I_{h} w=I_{h} w$, we obtain

$$
\begin{aligned}
\| w-P_{h} w||_{W^{1, p}} & \leq\left\|w-I_{h} w\right\|_{W^{1, p}}+\left\|I_{h} w-P_{h} w\right\|_{W^{1, p}} \\
& =\left\|w-I_{h} w\right\|_{W^{1, p}}+\left\|P_{h} I_{h} w-P_{h} w\right\|_{W^{1, p}} \\
& \leq C h^{k}|w|_{W^{k+1, p}}+\left\|I_{h} w-w\right\|_{W^{1, p}} \\
& \leq C h^{k}|w|_{W^{k+1, p}},
\end{aligned}
$$

which proves (2.9).

The independence of the constant $C$ in $p$ may be traced through the proof given in [12]. Alternatively, the independence of the constant $C$ in $p$ can be obtained through an interpolation argument we outline.

Let $1<p<\infty$ and $1 \leq q \leq \infty$. Using a notation similar to the one used in [9], we denote by $W^{k, p, q}(\Omega)$ the interpolation space $\left(W^{k, 1}(\Omega), W^{k, \infty}(\Omega)\right)_{1-1 / p, q, K}$ between $W^{k, 1}(\Omega)$ and $W^{k, \infty}(\Omega)$ as defined in [6, Definition 3.2.4]. The letter $K$, and also $K^{\prime}$ to be used below, refers to the function norm [6, (3.2.9)]. We note that it is assumed in [9] that the domain $\Omega$ is a minimally smooth domain, also known as a Lipschitz domain.

By [6, Theorem 3.2.23], $W^{k, p, q}(\Omega)$ is an exact interpolation space of order $\theta=$ $1-1 / p$ as defined in [6, Definition 3.2.22].

Moreover, by [6, Corollary 3.2.13 (a)], $W^{k, 2,2}(\Omega) \subset W^{k, 2, \infty}(\Omega)$. Thus since $W^{k, 2,2}(\Omega)$ is of order $\theta_{1}=1 / 2$ and $W^{k, \infty, \infty}(\Omega)$ is of order $\theta_{2}=1$, by [6, Proposition 3.2.16 (a)] and the reiteration theorem [6, Theorem 3.2.20],

$$
W^{k, p, q}(\Omega)=\left(W^{k, 2,2}(\Omega), W^{k, \infty, \infty}(\Omega)\right)_{1-2 / p, q, K^{\prime}}, \quad 1 \leq q \leq \infty .
$$

On the other hand, it is shown in $\left[9\right.$, p. 595] that $W^{k, p}(\Omega)=W^{k, p, p}(\Omega)$ with equivalent norms. It can be seen from [9, Theorem 1] that the constants in the norm equivalence are independent of $p$. We conclude that $W^{k, p / 2}(\Omega)$ is an exact interpolation space of order $1-2 / p$ between $W^{k, 2}(\Omega)$ and $W^{k, \infty}(\Omega)$. By [6, Definition 3.2.22], this means that since $P_{h}$ is a bounded linear map from $W^{k, 2}(\Omega)$ into itself with norm $M_{1}$, and also a bounded linear map from $W^{k, \infty}(\Omega)$ into itself with norm $M_{2}$, then $P_{h}$ is a bounded linear map from $W^{k, p / 2}(\Omega)$ into itself and its norm is bounded by $M_{1}^{1-2 / p} M_{2}^{2 / p}$, which is easily seen to be bounded above by a constant independent of $p$.

Lemma 2.2 Assume that Assumption 2.1 of elliptic regularity holds. Let $r \in L^{p}(\Omega), p \geq 2$ and let $v \in V_{h} \cap H_{0}^{1}(\Omega)$ solve

$$
\int_{\Omega}\left[\left(\operatorname{cof} D^{2} u\right) D v\right] \cdot D w d x=\int_{\Omega} r w d x, w \in V_{h} \cap H_{0}^{1}(\Omega) .
$$

Then

$$
\|v\|_{\widetilde{W}^{2, p}\left(\mathcal{T}_{h}\right)} \leq C p\|r\|_{L^{p}}
$$


Proof With these notation the solution $v$ of (2.10) is given by $v=P_{h} \phi$. Let $w \in V_{h} \cap H_{0}^{1}(\Omega)$. We have by (2.2) and (2.3)

$$
\begin{aligned}
\|v\|_{\widetilde{W}^{2, p}\left(\mathcal{T}_{h}\right)} & =\left\|P_{h} \phi\right\|_{\widetilde{W}^{2, p}\left(\mathcal{T}_{h}\right)} \leq\left\|P_{h} \phi-\phi\right\|_{\widetilde{W}^{2, p}\left(\mathcal{T}_{h}\right)}+\|\phi\|_{\widetilde{W}^{2, p}\left(\mathcal{T}_{h}\right)} \\
& \leq\left\|\phi-\tilde{I}_{h} \phi\right\|_{\widetilde{W}^{2, p}\left(\mathcal{T}_{h}\right)}+\left\|\tilde{I}_{h} \phi-P_{h} \phi\right\|_{\widetilde{W}^{2, p}\left(\mathcal{T}_{h}\right)}+\|\phi\|_{\widetilde{W}^{2, p}\left(\mathcal{T}_{h}\right)} \\
& \leq C\|\phi\|_{W^{2, p}}+C h^{-1}\left\|\tilde{I}_{h} \phi-P_{h} \phi\right\|_{W^{1, p}}+\|\phi\|_{\widetilde{W}^{2, p}\left(\mathcal{T}_{h}\right)} .
\end{aligned}
$$

By (2.9) and (2.4)

$$
\left\|\tilde{I}_{h} \phi-P_{h} \phi\right\|_{W^{1, p}} \leq\left\|\tilde{I}_{h} \phi-\phi\right\|_{W^{1, p}}+\left\|\phi-P_{h} \phi\right\|_{W^{1, p}} \leq C h\|\phi\|_{W^{2, p}} .
$$

Using (2.1) we conclude by elliptic regularity that

$$
\|v\|_{\widetilde{W}^{2, p}\left(\mathcal{T}_{h}\right)} \leq C\|\phi\|_{W^{2, p}} \leq C p\|r\|_{L^{p}}
$$

This proves (2.11).

Lemma 2.3 Let $r \in V_{h}$. Then for $p \geq 2$

$$
\|r\|_{L^{p}} \leq C \sup _{\substack{z \neq 0 \\ z \in V_{h}}} \frac{|(r, z)|}{\|z\|_{L^{q}}} \quad \frac{1}{p}+\frac{1}{q}=1 .
$$

Proof We have

$$
\|r\|_{L^{p}}=\sup _{\substack{w \neq 0 \\ w \in L^{q}}} \frac{|(r, w)|}{\|w\|_{L^{q}}} .
$$

Let $P_{V_{h}}$ be the $L^{2}$ projection into $V_{h}$. The projection is known to be stable in $L^{q}$ [10], i.e. for $w \in L^{q}(\Omega)$

$$
\left\|P_{V_{h}} w\right\|_{L^{q}} \leq C^{\theta}\|w\|_{L^{q}}, \theta=\left|1-\frac{2}{q}\right| .
$$

Since $p \geq 2,-1<1-2 / q \leq 2$ and hence the constant $C^{\theta}$ is bounded uniformly in $q$. Since $r \in V_{h},(r, w)=\left(r, P_{V_{h}} w\right)$ and therefore

$$
\frac{|(r, w)|}{\|w\|_{L^{q}}} \leq C \frac{\left|\left(r, P_{V_{h}} w\right)\right|}{\left\|P_{V_{h}} w\right\|_{L^{q}}} \leq C \sup _{\substack{z \neq 0 \\ z \in V_{h}}} \frac{|(r, z)|}{\|z\|_{L^{q}}} .
$$

This concludes the proof. 


\section{Error analysis of the mixed method with the elliptic regularity assumption}

For this erratum the mapping $T: V_{h} \times \Sigma_{h} \rightarrow V_{h} \times \Sigma_{h}$ is defined by

$$
T\left(w_{h}, \eta_{h}\right)=\left(T_{1}\left(w_{h}, \eta_{h}\right), T_{2}\left(w_{h}, \eta_{h}\right)\right)
$$

where $T_{1}\left(w_{h}, \eta_{h}\right)$ and $T_{2}\left(w_{h}, \eta_{h}\right)$ satisfy

$$
\begin{aligned}
&\left(\eta_{h}-T_{2}\left(w_{h}, \eta_{h}\right), \tau\right)+\left(\operatorname{div} \tau, D\left(w_{h}-T_{1}\left(w_{h}, \eta_{h}\right)\right)\right) \\
&-\left\langle D\left(w_{h}-T_{1}\left(w_{h}, \eta_{h}\right)\right), \tau n\right\rangle=\left(\eta_{h}, \tau\right) \\
&+\left(\operatorname{div} \tau, D w_{h}\right)-\left\langle D w_{h}, \tau n\right\rangle, \quad \forall \tau \in \Sigma_{h} \\
&\left(( \operatorname { c o f } D ^ { 2 } u ) D \left(w_{h}-\right.\right.\left.\left.T_{1}\left(w_{h}, \eta_{h}\right)\right), D v\right)=(f, v)-\left(\operatorname{det} \eta_{h}, v\right), \forall v \in V_{h} \cap H_{0}^{1}(\Omega) \\
& w_{h}-T_{1}\left(w_{h}, \eta_{h}\right)=0 \quad \text { on } \partial \Omega .
\end{aligned}
$$

It is shown in [3, Lemma 3.4] that a fixed point of (3.1)-(3.3) with $w_{h}=g_{h}$ on $\partial \Omega$ solves the nonlinear problem [1, (3)].

For this erratum we define

$$
\bar{B}_{h}(\rho)=\left\{\left(w_{h}, \eta_{h}\right) \in V_{h} \times \Sigma_{h},\left\|w_{h}-I_{h} u\right\|_{W^{2, \infty}\left(\mathcal{T}_{h}\right)} \leq \rho,\left\|\eta_{h}-I_{h} \sigma\right\|_{L^{\infty}} \leq \rho\right\} .
$$

Recall that $B_{h}(\rho)=\bar{B}_{h}(\rho) \cap Z_{h}$ with $Z_{h}$ defined on [1, p. 7].

Lemma 3.1 For a positive constant $C_{0}$ and $\rho=C_{0} h^{k-1}$, we have $B_{h}(\rho) \neq \emptyset$.

Proof It is shown in [3, Lemma 3.5] that there exists $\eta_{h} \in \Sigma_{h}$ such that $\left(I_{h} u, \eta_{h}\right) \in Z_{h}$. We estimate $\left\|\eta_{h}-I_{h} \sigma\right\|_{L^{\infty}}$. We have

$$
\left(\eta_{h}-I_{h} \sigma, \tau\right)=\left(\sigma-I_{h} \sigma, \tau\right)-\left(\operatorname{div} \tau, D\left(I_{h} u-u\right)\right)+\left\langle D\left(I_{h} u-u\right), \tau n\right\rangle .
$$

Let $p>1$ and $q$ such that $1 / p+1 / q=1$. We have by Lemma 2.3

$$
\left\|\eta_{h}-I_{h} \sigma\right\|_{L^{p}} \leq C \sup _{\substack{\tau \neq 0 \\ \tau \in \Sigma_{h}}} \frac{\left|\left(\eta_{h}-I_{h} \sigma, \tau\right)\right|}{\|\tau\|_{L^{q}}} .
$$

By Cauchy-Schwarz inequality, the scale-trace inequality (2.5), inverse estimates and approximation properties of $I_{h}$

$$
\begin{aligned}
\left|\left(\eta_{h}-I_{h} \sigma, \tau\right)\right| \leq & \left\|\sigma-I_{h} \sigma\right\|_{L^{p}}\|\tau\|_{L^{q}}+\left\|D\left(I_{h} u-u\right)\right\|_{L^{p}}\|\operatorname{div} \tau\|_{L^{q}} \\
& +C\left\|D\left(I_{h} u-u\right)\right\|_{L^{p}(\partial \Omega)}\|\tau\|_{L^{q}(\partial \Omega)} \\
\leq & \left\|\sigma-I_{h} \sigma\right\|_{L^{\infty}}\|\tau\|_{L^{q}}+C h^{-1}\left\|D\left(I_{h} u-u\right)\right\|_{L^{\infty}}\|\tau\|_{L^{q}} \\
& +C h^{-\frac{1}{2}}\left\|D\left(I_{h} u-u\right)\right\|_{L^{\infty}}\|\tau\|_{L^{q}} \\
\leq & \left(C h^{k+1}+C h^{k-1}\right)\|\tau\|_{L^{q}} \leq C h^{k-1}\|\tau\|_{L^{q}} .
\end{aligned}
$$


We conclude that $\left\|\eta_{h}-I_{h} \sigma\right\|_{L^{p}} \leq C h^{k-1}$. By an inverse estimate

$$
\left\|\eta_{h}-I_{h} \sigma\right\|_{L^{\infty}} \leq C h^{-\frac{2}{p}}\left\|\eta_{h}-I_{h} \sigma\right\|_{L^{p}} \leq C h^{-\frac{2}{p}} h^{k-1}
$$

Choosing $p$ such that $|\ln h| \leq p \leq 2|\ln h|$, we obtain $\left\|\eta_{h}-I_{h} \sigma\right\|_{L^{\infty}} \leq C h^{k-1}$. This concludes the proof.

Lemma 3.2 The mapping $T$ does not move the center $\left(I_{h} u, I_{h} \sigma\right)$ of the ball $\bar{B}_{h}(\rho)$ too far, i.e. for $h$ sufficiently small

$$
\begin{aligned}
\left\|I_{h} u-T_{1}\left(I_{h} u, I_{h} \sigma\right)\right\|_{W^{2, \infty}\left(\mathcal{T}_{h}\right)} & \leq C_{1} h^{k} \\
\left\|I_{h} \sigma-T_{2}\left(I_{h} u, I_{h} \sigma\right)\right\|_{L^{\infty}} & \leq C_{2} h^{k-1}
\end{aligned}
$$

Proof By [1, Lemma 2.1], on each element $K$

$$
\left\|\operatorname{det}\left(I_{h} \sigma\right)-\operatorname{det} \sigma\right\|_{L^{\infty}(K)} \leq C\left\|\frac{1}{2} I_{h} \sigma+\frac{1}{2} \sigma\right\|_{L^{\infty}(K)}\left\|I_{h} \sigma-\sigma\right\|_{L^{\infty}(K)}
$$

By approximation properties $\left\|I_{h} \sigma-\sigma\right\|_{L^{\infty}(K)} \leq C h^{k+1}$, so $\left\|I_{h} \sigma\right\|_{L^{\infty}} \leq C\|\sigma\|_{L^{\infty}}$, and

$$
\left\|\operatorname{det}\left(I_{h} \sigma\right)-\operatorname{det} \sigma\right\|_{L^{\infty}(K)} \leq C\left\|I_{h} \sigma-\sigma\right\|_{L^{\infty}(K)} \leq C h^{k+1} .
$$

By (3.2), (3.3), discrete elliptic regularity and (3.6)

$$
\begin{aligned}
\left\|I_{h} u-T_{1}\left(I_{h} u, I_{h} \sigma\right)\right\|_{\widetilde{W}^{2, p}\left(\mathcal{T}_{h}\right)} & \leq C p\left\|\operatorname{det} I_{h} \sigma-f\right\|_{L^{p}}=C\left\|\operatorname{det} I_{h} \sigma-\operatorname{det} D^{2} u\right\|_{L^{p}} \\
& \leq C p\left\|\operatorname{det} I_{h} \sigma-\operatorname{det} D^{2} u\right\|_{L^{\infty}} \leq C p h^{k+1} .
\end{aligned}
$$

Choosing $p$ such that $|\ln h| \leq p \leq 2|\ln h|$, we obtain by an inverse estimate

$$
\begin{aligned}
\left\|I_{h} u-T_{1}\left(I_{h} u, I_{h} \sigma\right)\right\|_{W^{2, \infty}\left(\mathcal{T}_{h}\right)} & \leq C h^{-\frac{2}{p}}\left\|I_{h} u-T_{1}\left(I_{h} u, I_{h} \sigma\right)\right\|_{W^{2, p}\left(\mathcal{T}_{h}\right)} \\
& \leq C h^{-\frac{2}{p}}\left\|I_{h} u-T_{1}\left(I_{h} u, I_{h} \sigma\right)\right\|_{\widetilde{W}^{2, p}\left(\mathcal{T}_{h}\right)} \\
& \leq C\left\|I_{h} u-T_{1}\left(I_{h} u, I_{h} \sigma\right)\right\|_{\widetilde{W}^{2, p}\left(\mathcal{T}_{h}\right)} \leq C h^{k+1}|\ln h| .
\end{aligned}
$$

We conclude that (3.4) holds.

Let $p>1$ and $q$ such that $1 / p+1 / q=1$. We have by Lemma 2.3

$$
\left\|I_{h} \sigma-T_{2}\left(I_{h} u, I_{h} \sigma\right)\right\|_{L^{p}} \leq C \sup _{\substack{\tau \neq 0 \\ \tau \in \Sigma_{h}}}\left|\left(I_{h} \sigma-T_{2}\left(I_{h} u, I_{h} \sigma\right), \tau\right)\right| /\|\tau\|_{L^{q}} .
$$

Moreover by (3.1) and using

$$
(\sigma, \tau)+(\operatorname{div} \tau, D u)-\langle D u, \tau n\rangle=0, \quad \forall \tau \in H^{1}(\Omega),
$$


we get

$$
\begin{aligned}
\left(I_{h} \sigma-T_{2}\left(I_{h} u, I_{h} \sigma\right), \tau\right)= & -\left(\operatorname{div} \tau, D\left(I_{h} u-T_{1}\left(I_{h} u, I_{h} \sigma\right)\right)\right) \\
& +\left\langle D\left(I_{h} u-T_{1}\left(I_{h} u, I_{h} \sigma\right)\right), \tau n\right\rangle \\
& +\left(I_{h} \sigma-\sigma, \tau\right)+\left(\operatorname{div} \tau, D\left(I_{h} u-u\right)\right) \\
& -\left\langle D\left(I_{h} u-u\right), \tau n\right\rangle .
\end{aligned}
$$

By Cauchy-Schwarz inequality, an inverse estimate, the trace-inverse inequality and approximation properties, we have

$$
\begin{aligned}
& \left|\left(I_{h} \sigma-\sigma, \tau\right)+\left(\operatorname{div} \tau, D\left(I_{h} u-u\right)\right)-\left\langle D\left(I_{h} u-u\right), \tau n\right\rangle\right| \leq\left(C h^{k+1}\|\sigma\|_{W^{k+1, \infty}}\right. \\
& \left.\quad+C h^{k-1}\|u\|_{W^{k+1, \infty}}+C h^{k-1}\|u\|_{W^{k+1, \infty}}\right)\|\tau\|_{L^{q}} \leq C h^{k-1}\|u\|_{W^{k+3, \infty}}\|\tau\|_{L^{q}} .
\end{aligned}
$$

Moreover

$$
\begin{aligned}
-(\operatorname{div} & \left.\tau, D\left(I_{h} u-T_{1}\left(I_{h} u, I_{h} \sigma\right)\right)\right)+\left\langle D\left(I_{h} u-T_{1}\left(I_{h} u, I_{h} \sigma\right)\right), \tau n\right\rangle \\
= & \sum_{K \in \mathcal{T}_{h}}\left(\tau, D^{2}\left(I_{h} u-T_{1}\left(I_{h} u, I_{h} \sigma\right)\right)\right)_{K}-\sum_{K \in \mathcal{T}_{h}}\left\langle D\left(I_{h} u-T_{1}\left(I_{h} u, I_{h} \sigma\right)\right), \tau n\right\rangle_{\partial K} \\
\quad & +\left\langle D\left(I_{h} u-T_{1}\left(I_{h} u, I_{h} \sigma\right)\right), \tau n\right\rangle .
\end{aligned}
$$

But by Cauchy-Schwarz inequality and the trace-inverse inequality

$$
\begin{aligned}
\mid & -\sum_{K \in \mathcal{T}_{h}}\left\langle D\left(I_{h} u-T_{1}\left(I_{h} u, I_{h} \sigma\right)\right), \tau n\right\rangle_{\partial K}+\left\langle D\left(I_{h} u-T_{1}\left(I_{h} u, I_{h} \sigma\right)\right), \tau n\right\rangle \mid \\
& \leq \sum_{K \in \mathcal{T}_{h}}\left|\left\langle D\left(I_{h} u-T_{1}\left(I_{h} u, I_{h} \sigma\right)\right), \tau n\right\rangle_{\partial K}\right| \\
& \leq \sum_{K \in \mathcal{T}_{h}}\left\|h^{-\frac{1}{q}} D\left(I_{h} u-T_{1}\left(I_{h} u, I_{h} \sigma\right)\right)\right\|_{L^{p}(\partial K)}\left\|h^{\frac{1}{q}} \tau n\right\|_{L^{q}(\partial K)} \\
& \leq C\left(\sum_{K \in \mathcal{T}_{h}} h^{-\frac{p}{q}}\left\|D\left(I_{h} u-T_{1}\left(I_{h} u, I_{h} \sigma\right)\right)\right\|_{L^{p}(\partial K)}^{p}\right)^{\frac{1}{p}}\|\tau\|_{L^{q}} .
\end{aligned}
$$

Therefore by Cauchy-Schwarz inequality

$$
\begin{aligned}
\mid- & \left(\operatorname{div} \tau, D\left(I_{h} u-T_{1}\left(I_{h} u, I_{h} \sigma\right)\right)\right)+\left\langle D\left(I_{h} u-T_{1}\left(I_{h} u, I_{h} \sigma\right)\right), \tau n\right\rangle \mid \\
\leq & C\left(\left\|I_{h} u-T_{1}\left(I_{h} u, I_{h} \sigma\right)\right\|_{W^{2, p}\left(\mathcal{T}_{h}\right)}\right. \\
& \left.\quad+\left(\sum_{K \in \mathcal{T}_{h}} h^{1-p}\left\|D\left(I_{h} u-T_{1}\left(I_{h} u, I_{h} \sigma\right)\right)\right\|_{L^{p}(\partial K)}^{p}\right)^{\frac{1}{p}}\right)\|\tau\|_{L^{q}} .
\end{aligned}
$$


We conclude from (3.7), (3.8), (3.9) and (3.10) that

$$
\left|\left(I_{h} \sigma-T_{2}\left(I_{h} u, I_{h} \sigma\right), \tau\right)\right| \leq\left(C h^{k-1}+C\left\|I_{h} u-T_{1}\left(I_{h} u, I_{h} \sigma\right)\right\|_{\widetilde{W}^{2, p}\left(\mathcal{T}_{h}\right)}\right)\|\tau\|_{L^{q}} .
$$

Thus using (2.1)

$$
\begin{aligned}
\left|\left(I_{h} \sigma-T_{2}\left(I_{h} u, I_{h} \sigma\right), \tau\right)\right| & \leq\left(C h^{k-1}+C\left\|I_{h} u-T_{1}\left(I_{h} u, I_{h} \sigma\right)\right\|_{W^{2, p}\left(\mathcal{T}_{h}\right)}\right)\|\tau\|_{L^{q}} \\
& \leq\left(C h^{k-1}+C\left\|I_{h} u-T_{1}\left(I_{h} u, I_{h} \sigma\right)\right\|_{W^{2, \infty}\left(\mathcal{T}_{h}\right)}\right)\|\tau\|_{L^{q}} .
\end{aligned}
$$

Choosing $p$ such that $|\ln h| \leq p \leq 2|\ln h|$ and using (3.7), we obtain

$$
\left\|I_{h} \sigma-T_{2}\left(I_{h} u, I_{h} \sigma\right)\right\|_{L^{\infty}} \leq C h^{-\frac{2}{p}}\left\|I_{h} \sigma-T_{2}\left(I_{h} u, I_{h} \sigma\right)\right\|_{L^{p}} \leq C h^{-\frac{2}{p}} h^{k-1} \leq C h^{k-1} .
$$

This concludes the proof.

Lemma 3.3 Let $\rho>0$ and $\left(w_{1}, \eta_{1}\right)$ and $\left(w_{2}, \eta_{2}\right)$ in $B_{h}(\rho)$. We have

$$
\left\|T_{2}\left(w_{1}, \eta_{1}\right)-T_{2}\left(w_{2}, \eta_{2}\right)\right\|_{L^{\infty}} \leq C_{3}\left\|T_{1}\left(w_{1}, \eta_{1}\right)-T_{1}\left(w_{2}, \eta_{2}\right)\right\|_{W^{2, \infty}\left(\mathcal{T}_{h}\right)},
$$

for a constant $C_{3} \geq 1$.

Proof For $\left(w_{1}, \eta_{1}\right)$ and $\left(w_{2}, \eta_{2}\right)$ in $B_{h}(\rho)$. We have using (3.1)

$$
\begin{aligned}
\left(T_{2}\left(w_{1}, \eta_{1}\right)-T_{2}\left(w_{2}, \eta_{2}\right), \tau\right)= & -\left(\operatorname{div} \tau, D\left(T_{1}\left(w_{1}, \eta_{1}\right)-T_{1}\left(w_{2}, \eta_{2}\right)\right)\right) \\
& +\left\langle D\left(T_{1}\left(w_{1}, \eta_{1}\right)-T_{1}\left(w_{2}, \eta_{2}\right)\right), \tau n\right\rangle \\
= & \sum_{K \in \mathcal{T}_{h}}\left(\tau, D^{2}\left(T_{1}\left(w_{1}, \eta_{1}\right)-T_{1}\left(w_{2}, \eta_{2}\right)\right)_{K}\right. \\
& -\sum_{K \in \mathcal{T}_{h}}\left\langle\tau n, D\left(T_{1}\left(w_{1}, \eta_{1}\right)-T_{1}\left(w_{2}, \eta_{2}\right)\right)\right\rangle_{\partial K} \\
& +\left\langle D\left(T_{1}\left(w_{1}, \eta_{1}\right)-T_{1}\left(w_{2}, \eta_{2}\right)\right), \tau n\right\rangle .
\end{aligned}
$$

Let $p \geq 2$ and $q$ such that $1 / p+1 / q=1$. We have

$$
\begin{aligned}
\mid \sum_{K \in \mathcal{T}_{h}}\left(\tau, D^{2}\left(T_{1}\left(w_{1}, \eta_{1}\right)-T_{1}\left(w_{2}, \eta_{2}\right)\right)_{K} \mid \leq\right. & C \| T_{1}\left(w_{1}, \eta_{1}\right) \\
& -T_{1}\left(w_{2}, \eta_{2}\right)\left\|_{W^{2, p}\left(\mathcal{T}_{h}\right)}\right\| \tau \|_{L^{q}} \\
\leq & C \| T_{1}\left(w_{1}, \eta_{1}\right) \\
& -T_{1}\left(w_{2}, \eta_{2}\right)\left\|_{W^{2, \infty}\left(\mathcal{T}_{h}\right)}\right\| \tau \|_{L^{q}} .
\end{aligned}
$$


Moreover by Cauchy-Schwarz and the scale-trace inequality (2.5)

$$
\begin{aligned}
\mid & -\sum_{K \in \mathcal{T}_{h}}\left\langle\tau n, D\left(T_{1}\left(w_{1}, \eta_{1}\right)-T_{1}\left(w_{2}, \eta_{2}\right)\right)\right\rangle_{\partial K}+\left\langle D\left(T_{1}\left(w_{1}, \eta_{1}\right)-T_{1}\left(w_{2}, \eta_{2}\right)\right), \tau n\right\rangle \mid \\
\leq & \sum_{K \in \mathcal{T}_{h}}\left|\left\langle\tau n, D\left(T_{1}\left(w_{1}, \eta_{1}\right)-T_{1}\left(w_{2}, \eta_{2}\right)\right)\right\rangle_{\partial K}\right| \\
= & \sum_{K \in \mathcal{T}_{h}}\left|\left\langle h^{\frac{1}{q}} \tau n, h^{-\frac{1}{q}} D\left(T_{1}\left(w_{1}, \eta_{1}\right)-T_{1}\left(w_{2}, \eta_{2}\right)\right)\right\rangle_{\partial K}\right| \leq C\|\tau\|_{L^{q}} \\
& \times\left(\sum_{K \in \mathcal{T}_{h}} h^{-\frac{p}{q}}\left\|D\left(T_{1}\left(w_{1}, \eta_{1}\right)-T_{1}\left(w_{2}, \eta_{2}\right)\right)\right\|_{L^{2}(\partial K)}^{p}\right)^{\frac{1}{p}} .
\end{aligned}
$$

Since $-p / q=1-p$ we obtain

$$
\left|\left(T_{2}\left(w_{1}, \eta_{1}\right)-T_{2}\left(w_{2}, \eta_{2}\right), \tau\right)\right| \leq C|| T_{1}\left(w_{1}, \eta_{1}\right)-T_{1}\left(w_{2}, \eta_{2}\right)\left\|_{\widetilde{W}^{2, p}\left(\mathcal{T}_{h}\right)}\right\| \tau \|_{L^{q}}
$$

And thus using (2.1)

$$
\begin{aligned}
\left|\left(T_{2}\left(w_{1}, \eta_{1}\right)-T_{2}\left(w_{2}, \eta_{2}\right), \tau\right)\right| & \leq C\left\|T_{1}\left(w_{1}, \eta_{1}\right)-T_{1}\left(w_{2}, \eta_{2}\right)\right\|_{W^{2, p}\left(\mathcal{T}_{h}\right)}\|\tau\|_{L^{q}} \\
& \leq C\left\|T_{1}\left(w_{1}, \eta_{1}\right)-T_{1}\left(w_{2}, \eta_{2}\right)\right\|_{W^{2, \infty}\left(\mathcal{T}_{h}\right)}\|\tau\|_{L^{q}}
\end{aligned}
$$

We conclude that

$$
\begin{aligned}
\left\|I_{h} \sigma-T_{2}\left(I_{h} u, I_{h} \sigma\right)\right\|_{L^{\infty}} & \leq C h^{-\frac{2}{p}}\left\|I_{h} \sigma-T_{2}\left(I_{h} u, I_{h} \sigma\right)\right\|_{L^{p}} \\
& \leq C h^{-\frac{2}{p}} \sup _{\substack{\tau \neq 0 \\
\tau \in \Sigma_{h}}}\left|\left(I_{h} \sigma-T_{2}\left(I_{h} u, I_{h} \sigma\right), \tau\right)\right| /\|\tau\|_{L^{q}} \\
& \leq C h^{-\frac{2}{p}}\left\|T_{1}\left(w_{1}, \eta_{1}\right)-T_{1}\left(w_{2}, \eta_{2}\right)\right\|_{W^{2, \infty}\left(\mathcal{T}_{h}\right)} \\
& \leq C\left\|T_{1}\left(w_{1}, \eta_{1}\right)-T_{1}\left(w_{2}, \eta_{2}\right)\right\|_{W^{2, \infty}\left(\mathcal{T}_{h}\right)},
\end{aligned}
$$

where we used Lemma 2.3 and choose $p$ such that $|\ln h| \leq p \leq 2|\ln h|$. This concludes the proof.

For $\left(w_{h}, \eta_{h}\right) \in Z_{h}$ we define

$$
\Gamma=\left(\left(\operatorname{cof} D^{2} u\right): \eta_{h}, v\right)+\left(\left(\operatorname{cof} D^{2} u\right) D w_{h}, D v\right) .
$$

We have the following analogue of [3, Lemma 3.7]

Lemma 3.4 Let $\left(w_{h}, \eta_{h}\right) \in Z_{h}$. Then

$$
\left|\left(\left(\operatorname{cof} D^{2} u\right): \eta_{h}, v\right)+\left(\left(\operatorname{cof} D^{2} u\right) D w_{h}, D v\right)\right| \leq C h^{\frac{1}{q}}\left\|w_{h}\right\|_{\widetilde{W}^{2, p}\left(\mathcal{T}_{h}\right)}\|v\|_{L^{q}},
$$

for all $v \in V_{h} \cap H_{0}^{1}(\Omega)$ and $p \geq 2,1 / p+1 / q=1$. 
Proof Denote by $P_{\Sigma_{h}}$ the $L^{2}$ projection into the space $\Sigma_{h}$. Put $A=\operatorname{cof} D^{2} u$. It is proven in the proof of [3, Lemma 3.7] that for $v \in V_{h} \cap H_{0}^{1}(\Omega)$

$$
\Gamma=-\sum_{K \in \mathcal{T}_{h}}\left(\operatorname{div}\left(P_{\Sigma_{h}}(v A)-v A\right), D w_{h}\right)_{K}+\left\langle\left(P_{\Sigma_{h}}(v A)-v A\right) n, D w_{h}\right\rangle_{\partial \Omega}
$$

We have

$$
\begin{aligned}
\Gamma= & \sum_{K \in \mathcal{T}_{h}}\left(P_{\Sigma_{h}}(v A)-v A, D^{2} w_{h}\right)_{K}-\sum_{K \in \mathcal{T}_{h}}\left\langle\left(P_{\Sigma_{h}}(v A)-v A\right) n, D w_{h}\right\rangle_{\partial K} \\
& +\left\langle\left(P_{\Sigma_{h}}(v A)-v A\right) n, D w_{h}\right\rangle_{\partial \Omega} .
\end{aligned}
$$

By Cauchy-Schwarz inequality

$$
\left|\sum_{K \in \mathcal{T}_{h}}\left(P_{\Sigma_{h}}(v A)-v A, D^{2} w_{h}\right)_{K}\right| \leq\left\|P_{\Sigma_{h}}(v A)-v A\right\|_{L^{q}}\left\|w_{h}\right\|_{W^{2, p}\left(\mathcal{T}_{h}\right)},
$$

and by Cauchy-Schwarz and the trace inequalities

$$
\begin{aligned}
\mid & -\sum_{K \in \mathcal{T}_{h}}\left\langle\left(P_{\Sigma_{h}}(v A)-v A\right) n, D w_{h}\right\rangle_{\partial K}+\left\langle\left(P_{\Sigma_{h}}(v A)-v A\right) n, D w_{h}\right\rangle_{\partial \Omega} \mid \\
& \leq \sum_{K \in \mathcal{T}_{h}}\left|\left\langle\left(P_{\Sigma_{h}}(v A)-v A\right) n, D w_{h}\right\rangle_{\partial K}\right| \\
& =\sum_{K \in \mathcal{T}_{h}}\left|\left\langle h^{\frac{1}{q}}\left(P_{\Sigma_{h}}(v A)-v A\right) n, h^{-\frac{1}{q}} D w_{h}\right\rangle_{\partial K}\right| \\
\leq & C \sum_{K \in \mathcal{T}_{h}}\left\|h^{\frac{1}{q}}\left(P_{\Sigma_{h}}(v A)-v A\right)\right\|_{L^{q}(\partial K)}\left\|h^{-\frac{1}{q}} D w_{h}\right\|_{L^{p}(\partial K)} \\
\leq & C h^{\frac{1}{q}}\left\|P_{\Sigma_{h}}(v A)-v A\right\|_{W^{1, q}}\left(\sum_{K \in \mathcal{T}_{h}} h^{1-p}\left\|D w_{h}\right\|_{L^{p}(\partial K)}^{p}\right)^{\frac{1}{p}} .
\end{aligned}
$$

Arguing as in the proof of [14, Lemma 4.4] we have for $m=0,1$

$$
\left\|P_{\Sigma_{h}}(v A)-v A\right\|_{W^{m, q}} \leq C h^{1-m}\|v\|_{L^{q}} .
$$

This follows from the stability in $L^{q}$ and $W^{1, q}$ of the $L^{2}$ projection [8], i.e. for $v \in W^{m, q}(\Omega), v=0$ on $\partial \Omega,\left\|P_{\Sigma_{h}}(v A)\right\|_{W^{m, q}} \leq C\|v A\|_{W^{m, q}}$.

As in the proof of Lemma 2.3, the constant in the $L^{q}$ stability of the $L^{2}$ projection is independent of $q$. For the $W^{1, q}$ stability, the independence in $q$ of the constant is obtained by tracing constants in the proof of [8, Theorem 4 and Theorem 3]. More precisely, constants in the interpolation estimates and inverse estimates used therein are independent of $q$, c.f. Sect. 2. In addition, the constant $\alpha$ in [8] is equal to 1 for quasi uniform triangulations, making the constants in the estimates independent of $q$. 
Since $P_{\Sigma_{h}} I_{h}(v A)=I_{h}(v A)$,

$$
\begin{aligned}
\left\|P_{\Sigma_{h}}(v A)-v A\right\|_{W^{m, q}} & \leq\left\|P_{\Sigma_{h}}(v A)-I_{h}(v A)\right\|_{W^{m, q}}+\left\|I_{h}(v A)-v A\right\|_{W^{m, q}} \\
& =\left\|P_{\Sigma_{h}}(v A)-P_{\Sigma_{h}} I_{h}(v A)\right\|_{W^{m, q}}+\left\|I_{h}(v A)-v A\right\|_{W^{m, q}} \\
& \leq C\left\|I_{h}(v A)-v A\right\|_{W^{m, q}} \leq C h^{k+1-m}\|v\|_{W^{k+1, q}} \\
& =C h^{k+1-m}\|v\|_{W^{k, q}} \leq C h^{1-m}\|v\|_{L^{2}},
\end{aligned}
$$

where in the last steps, we note that $v$ is a piecewise polynomial of degree $k$ and use an inverse estimate. It therefore follows from (3.14)-(3.16) that (3.13) holds.

The mapping $T_{1}$ has a fixed contraction property, i.e.

Lemma 3.5 For $h$ sufficiently small, we have for $\left(w_{1}, \eta_{1}\right)$ and $\left(w_{2}, \eta_{2}\right)$ in $B_{h}(\rho)$

$$
\begin{aligned}
\left\|T_{1}\left(w_{1}, \eta_{1}\right)-T_{1}\left(w_{2}, \eta_{2}\right)\right\|_{W^{2, \infty}\left(\mathcal{T}_{h}\right)} \leq & \frac{1}{4 C_{3}}\left\|w_{1}-w_{2}\right\|_{W^{2, \infty}\left(\mathcal{T}_{h}\right)} \\
& +\left(\frac{1}{4 C_{3}}+C|\ln h| \rho\right)\left\|\eta_{1}-\eta_{2}\right\|_{L^{\infty}}
\end{aligned}
$$

Proof The proof is a variant of [3, Lemma 3.10] and [3, Lemma 3.11]. Using (3.2) we have

$$
\begin{aligned}
& \left(\left(\operatorname{cof} D^{2} u\right) D\left(T_{1}\left(w_{1}, \eta_{1}\right)-T_{1}\left(w_{2}, \eta_{2}\right)\right), D v\right)=\left(\left(\operatorname{cof} D^{2} u\right) D\left(w_{1}-w_{2}\right), D v\right) \\
& \quad+\left(\operatorname{det} \eta_{1}-\operatorname{det} \eta_{2}, v\right)+\left(\left(\operatorname{cof} D^{2} u\right):\left(\eta_{1}-\eta_{2}\right), v\right)-\left(\left(\operatorname{cof} D^{2} u\right):\left(\eta_{1}-\eta_{2}\right), v\right)
\end{aligned}
$$

for all $v \in V_{h}$. Using the definition of $\Gamma$, (3.12) with $w_{h}=w_{1}-w_{2}, \eta_{h}=\eta_{1}-\eta_{2}$, and Lemma 3.4, we have

$$
\begin{aligned}
\left(\left(\operatorname{cof} D^{2} u\right) D\left(T_{1}\left(w_{1}, \eta_{1}\right)-T_{1}\left(w_{2}, \eta_{2}\right)\right), D v\right)= & -\left(\left(\operatorname{cof} D^{2} u\right):\left(\eta_{1}-\eta_{2}\right), v\right) \\
& +\left(\operatorname{det} \eta_{1}-\operatorname{det} \eta_{2}, v\right)+\Gamma,
\end{aligned}
$$

for all $v \in V_{h}$ with

$$
|\Gamma| \leq h^{\frac{1}{q}}\left\|w_{h}\right\|_{\widetilde{W}^{2, p}\left(\mathcal{T}_{h}\right)}\|v\|_{L^{q}}
$$

with $p \geq 2,1 / p+1 / q=1$.

By [1, Lemma 1], on each element $K$ we have

$$
\operatorname{det} \eta_{1}-\operatorname{det} \eta_{2}=\operatorname{cof}\left(\frac{1}{2} \eta_{1}+\frac{1}{2} \eta_{2}\right):\left(\eta_{1}-\eta_{2}\right)
$$


Therefore on each element $K$

$$
\begin{aligned}
& \left(\operatorname{cof} D^{2} u\right):\left(\eta_{1}-\eta_{2}\right)-\left(\operatorname{det} \eta_{1}-\operatorname{det} \eta_{2}\right) \\
& \quad=\left(\left(\operatorname{cof} D^{2} u\right)-\operatorname{cof}\left(\frac{1}{2} \eta_{1}+\frac{1}{2} \eta_{2}\right):\left(\eta_{1}-\eta_{2}\right)\right. \\
& \quad=\operatorname{cof}\left(D^{2} u-\frac{1}{2} \eta_{1}-\frac{1}{2} \eta_{2}\right):\left(\eta_{1}-\eta_{2}\right) .
\end{aligned}
$$

Let us define

$$
A=\left(\operatorname{cof} \sigma-\frac{1}{2} \eta_{1}-\frac{1}{2} \eta_{2}\right):\left(\eta_{1}-\eta_{2}\right)
$$

We have

$$
\begin{aligned}
\sigma-\left(\frac{1}{2} \eta_{1}+\frac{1}{2} \eta_{2}\right) & =\sigma-I_{h} \sigma+\frac{1}{2} I_{h} \sigma+\frac{1}{2} I_{h} \sigma-\left(\frac{1}{2} \eta_{1}+\frac{1}{2} \eta_{2}\right) \\
& =\sigma-I_{h} \sigma+\frac{1}{2}\left(I_{h} \sigma-\eta_{1}\right)+\frac{1}{2}\left(I_{h} \sigma-\eta_{2}\right) .
\end{aligned}
$$

We conclude that

$$
\begin{aligned}
\left\|\sigma-\left(\frac{1}{2} \eta_{1}+\frac{1}{2} \eta_{2}\right)\right\|_{L^{\infty}(K)} & \leq\left\|\sigma-I_{h} \sigma\right\|_{L^{\infty}(K)} \\
& +\frac{1}{2}\left\|I_{h} \sigma-\eta_{1}\right\|_{L^{\infty}(K)}+\frac{1}{2}\left\|I_{h} \sigma-\eta_{2}\right\|_{L^{\infty}(K)} \\
& \leq C h^{k+1}+C \rho .
\end{aligned}
$$

It follows from (3.21) that

$$
\left\|\left(\operatorname{cof} D^{2} u\right):\left(\eta_{1}-\eta_{2}\right)-\left(\operatorname{det} \eta_{1}-\operatorname{det} \eta_{2}\right)\right\|_{L^{p}} \leq\left(C h^{k+1}+C \rho\right)\left\|\eta_{1}-\eta_{2}\right\|_{L^{\infty}}
$$

Let us define the linear form $L$ on $V_{h}$ by

$$
L(v)=\left(\left(\operatorname{cof} D^{2} u\right) D\left(T_{1}\left(w_{1}, \eta_{1}\right)-T_{1}\left(w_{2}, \eta_{2}\right)\right), D v\right)
$$

By the Riesz representation theorem, there exists $r \in V_{h}$ with $L(v)=(r, v)$ for all $v \in V_{h}$. Moreover by Lemma $2.3\|r\|_{L^{p}} \leq C \sup |L(v)| /\|v\|_{L^{q}}$. We conclude from (3.19), (3.20) and (3.22) that

$$
\begin{aligned}
& v \neq 0 \\
& v \in V_{h}
\end{aligned}
$$

$$
\|r\|_{L^{p}} \leq C h^{\frac{1}{q}}\left\|w_{1}-w_{2}\right\|_{\widetilde{W}^{2, p}\left(\mathcal{T}_{h}\right)}+\left(C h^{k+1}+C \rho\right)\left\|\eta_{1}-\eta_{2}\right\|_{L^{\infty}} .
$$


By discrete elliptic regularity and (2.1)

$$
\begin{aligned}
\left\|T_{1}\left(w_{1}, \eta_{1}\right)-T_{1}\left(w_{2}, \eta_{2}\right)\right\|_{\widetilde{W}^{2, p}\left(\mathcal{T}_{h}\right) \leq} & C p h^{\frac{1}{q}}\left\|w_{1}-w_{2}\right\|_{\widetilde{W}^{2, p}\left(\mathcal{T}_{h}\right)} \\
& +\left(C h^{k+1}+C \rho\right) p\left\|\eta_{1}-\eta_{2}\right\|_{L^{\infty}} \\
\leq & C p h^{\frac{1}{q}}\left\|w_{1}-w_{2}\right\|_{W^{2, \infty}\left(\mathcal{T}_{h}\right)} \\
& +\left(C h^{k+1}+C \rho\right) p\left\|\eta_{1}-\eta_{2}\right\|_{L^{\infty}}
\end{aligned}
$$

Since $p \geq 2$ and $0<h \leq 1$, we have $h^{1 / q} \leq h^{1 / 2}$. Choosing $p$ such that $|\ln h| \leq p \leq$ $2|\ln h|$ we have $p h^{1 / 2} \leq C|\ln h| h^{1 / 2} \leq 1 /\left(4 C_{3}\right)$ for $h$ sufficiently small. Similarly $C h^{k+1}|\ln h| \leq 1 /\left(4 C_{3}\right)$ for $h$ sufficiently small. We conclude using an inverse estimate that

$$
\begin{aligned}
\left\|T_{1}\left(w_{1}, \eta_{1}\right)-T_{1}\left(w_{2}, \eta_{2}\right)\right\|_{W^{2, \infty}\left(\mathcal{T}_{h}\right)} \leq & C h^{-\frac{2}{p}}\left\|T_{1}\left(w_{1}, \eta_{1}\right)-T_{1}\left(w_{2}, \eta_{2}\right)\right\|_{W^{2, p}\left(\mathcal{T}_{h}\right)} \\
\leq & C h^{-\frac{2}{p}}\left\|T_{1}\left(w_{1}, \eta_{1}\right)-T_{1}\left(w_{2}, \eta_{2}\right)\right\|_{\widetilde{W}^{2, p}\left(\mathcal{T}_{h}\right)} \\
\leq & C\left\|T_{1}\left(w_{1}, \eta_{1}\right)-T_{1}\left(w_{2}, \eta_{2}\right)\right\|_{\widetilde{W}^{2, p}\left(\mathcal{T}_{h}\right)} \\
\leq & \frac{1}{4 C_{3}}\left\|w_{1}-w_{2}\right\|_{W^{2, \infty}\left(\mathcal{T}_{h}\right)} \\
& +\left(\frac{1}{4 C_{3}}+C|\ln h| \rho\right)\left\|\eta_{1}-\eta_{2}\right\|_{L^{\infty}} .
\end{aligned}
$$

This completes the proof.

Lemma 3.6 Let $\rho(h)=2 C_{4} h^{k-1}$ where $C_{4}=\max \left(C_{0}, C_{1}, 2 C_{2}\right)$ with $C_{0}$ the constant in Lemma 3.1 and $C_{1}, C_{2}$ the constants from Lemma 3.2. Then $T$ maps $B_{h}(\rho)$ into itself for $h$ sufficiently small.

Proof Let $\left(w_{h}, \eta_{h}\right) \in B_{h}(\rho)$. By definition, $\left\|w_{h}-I_{h} u\right\|_{W^{2, \infty}\left(\mathcal{T}_{h}\right)} \leq \rho$ and $\| \eta_{h}-$ $I_{h} \sigma \|_{L^{\infty}} \leq \rho$. By (3.18) and (3.4), for $h$ sufficiently small

$$
\begin{aligned}
\left\|T_{1}\left(w_{h}, \eta_{h}\right)-I_{h} u\right\|_{W^{2, \infty}\left(\mathcal{T}_{h}\right) \leq} & \left\|T_{1}\left(w_{h}, \eta_{h}\right)-T_{1}\left(I_{h} u, I_{h} \sigma\right)\right\|_{W^{2, \infty}\left(\mathcal{T}_{h}\right)} \\
& +\left\|T_{1}\left(I_{h} u, I_{h} \sigma\right)-I_{h} u\right\|_{W^{2, \infty}\left(\mathcal{T}_{h}\right)} \\
\leq & \left(\frac{1}{4}+C|\ln h| h^{k-1}\right)\left\|\eta_{h}-I_{h} \sigma\right\|_{L^{\infty}} \\
& +\frac{1}{4}\left\|w_{h}-I_{h} u\right\|_{\widetilde{H}^{2}\left(\mathcal{T}_{h}\right)}+C_{1} h^{k} \\
\leq & \frac{3 \rho}{4}+C_{1} h^{k}=\frac{3 \rho}{4}+\frac{C_{1} h}{2 C_{5}} \rho \leq \rho .
\end{aligned}
$$

In addition, by (3.18), (3.11) and (3.5) and a similar argument we get

$$
\begin{aligned}
& \left\|T_{2}\left(w_{h}, \eta_{h}\right)-I_{h} \sigma\right\|_{L^{\infty}} \\
& \quad \leq\left\|T_{2}\left(w_{h}, \eta_{h}\right)-T_{2}\left(I_{h} u, I_{h} \sigma\right)\right\|_{L^{\infty}}+\left\|T_{2}\left(I_{h} u, I_{h} \sigma\right)-I_{h} \sigma\right\|_{L^{\infty}}
\end{aligned}
$$




$$
\begin{aligned}
\leq & C_{3}\left\|T_{1}\left(w_{h}, \eta_{h}\right)-T_{1}\left(I_{h} u, I_{h} \sigma\right)\right\|_{W^{2, \infty}\left(\mathcal{T}_{h}\right)}+\left\|T_{2}\left(I_{h} u, I_{h} \sigma\right)-I_{h} \sigma\right\|_{L^{\infty}} \\
\leq & \frac{1}{4}\left\|\eta_{h}-I_{h} \sigma\right\|_{L^{\infty}}+C|\ln h| \rho\left\|\eta_{h}-I_{h} \sigma\right\|_{L^{\infty}} \\
& +\frac{1}{4}\left\|w_{h}-I_{h} u\right\|_{W^{2, \infty}\left(\mathcal{T}_{h}\right)}+C_{2} h^{k-1} \\
\leq & \rho
\end{aligned}
$$

for $h$ sufficiently small. By (3.1) $\left(T_{1}\left(w_{h}, \eta_{h}\right), T_{2}\left(w_{h}, \eta_{h}\right)\right)$ is in the space $Z_{h}$. This concludes the proof.

We can now claim

Theorem 3.7 Let $(u, \sigma) \in H^{k+3}(\Omega) \times H^{k+1}(\Omega)^{d \times d}$ denotes the unique convex solution of $[1,(1)]$ with $k \geq 2$. Then the problem [1, (3)] has a unique solution in $B_{h}(\rho) \subset V_{h} \times \Sigma_{h}$ for $h$ sufficiently small and with $\rho(h)$ given in Lemma 3.6.

Proof The proof follows from the Brouwer fixed point theorem. For $h$ sufficiently small and for $\left(w_{1}, \eta_{1}\right),\left(w_{2}, \eta_{2}\right) \in B_{h}(\rho)$, by (3.18) and (3.11)

$$
\begin{aligned}
& \left\|T_{1}\left(w_{1}, \eta_{1}\right)-T_{1}\left(w_{2}, \eta_{2}\right)\right\|_{W^{2, \infty}\left(\mathcal{T}_{h}\right)}+\left\|T_{2}\left(w_{1}, \eta_{1}\right)-T_{2}\left(w_{2}, \eta_{2}\right)\right\|_{L^{\infty}} \\
& \quad \leq C\left\|T_{1}\left(w_{1}, \eta_{1}\right)-T_{1}\left(w_{2}, \eta_{2}\right)\right\|_{W^{2, \infty}\left(\mathcal{T}_{h}\right)} \\
& \quad \leq C\left\|w_{1}-w_{2}\right\|_{W^{2, \infty}\left(\mathcal{T}_{h}\right)}+C\left\|\eta_{1}-\eta_{2}\right\|_{L^{\infty}}
\end{aligned}
$$

Hence the mapping $T$ is continuous in $B_{h}(\rho)$. Since for $h$ sufficiently small and the choice of $\rho(h)$, the continuous mapping $T$ maps the closed ball $B_{h}(\rho)$ into itself, there exists a fixed point $\left(u_{h}, \sigma_{h}\right)$ in $B_{h}(\rho)$.

Assume that $\left(w_{h}^{1}, \eta_{h}^{1}\right)$ and $\left(w_{h}^{2}, \eta_{h}^{2}\right)$ are two fixed points of $T$. Then $T_{1}\left(w_{h}^{1}, \eta_{h}^{1}\right)=w_{h}^{1}$ and $T_{1}\left(w_{h}^{2}, \eta_{h}^{2}\right)=w_{h}^{2}$. By (3.18) we have

$$
\left\|w_{h}^{1}-w_{h}^{2}\right\|_{W^{2, \infty}\left(\mathcal{T}_{h}\right)} \leq \frac{1}{2 C_{3}}\left\|\eta_{h}^{1}-\eta_{h}^{2}\right\|_{L^{\infty}}+\frac{1}{4}\left\|w_{h}^{1}-w_{h}^{2}\right\|_{W^{2, \infty}\left(\mathcal{T}_{h}\right)}
$$

and so

$$
\left\|w_{h}^{1}-w_{h}^{2}\right\|_{W^{2, \infty}\left(\mathcal{T}_{h}\right)} \leq \frac{2}{3 C_{3}}\left\|\eta_{h}^{1}-\eta_{h}^{2}\right\|_{L^{\infty}}
$$

We also have $T_{2}\left(w_{h}^{1}, \eta_{h}^{1}\right)=\eta_{h}^{1}$ and $T_{2}\left(w_{h}^{2}, \eta_{h}^{2}\right)=\eta_{h}^{2}$. By (3.11)

$$
\left\|\eta_{h}^{1}-\eta_{h}^{2}\right\|_{L^{\infty}} \leq C_{3}\left\|w_{h}^{1}-w_{h}^{2}\right\|_{W^{2, \infty}\left(\mathcal{T}_{h}\right)} \leq \frac{2}{3}\left\|\eta_{h}^{1}-\eta_{h}^{2}\right\|_{L^{2}}
$$

This implies $\eta_{h}^{1}=\eta_{h}^{2}$ and so $w_{h}^{1}=w_{h}^{2}$. This proves uniqueness.

The following error estimates hold 
Theorem 3.8 Under the assumptions of Theorem 3.7, the solution $\left(u_{h}, \sigma_{h}\right)$ of (3.1)(3.3) satisfies

$$
\begin{aligned}
\left\|u-u_{h}\right\|_{W^{2, \infty}\left(\mathcal{T}_{h}\right)} & \leq C h^{k-1} \\
\left\|\sigma-\sigma_{h}\right\|_{L^{\infty}} & \leq C h^{k-1} .
\end{aligned}
$$

Proof By the definition of the ball $B_{h}(\rho)$, the existence of the solution $\left(u_{h}, \sigma_{h}\right)$ in $B_{h}(\rho)$ with $\rho=O\left(h^{k-1}\right)$ given in Theorem 3.7, we have

$$
\begin{aligned}
\left\|I_{h} u-u_{h}\right\|_{W^{2, \infty}\left(\mathcal{T}_{h}\right)} & \leq C h^{k-1} \\
\left\|I_{h} \sigma-\sigma_{h}\right\|_{L^{\infty}} & \leq C h^{k-1} .
\end{aligned}
$$

The estimates (3.23) and (3.24) then follow from triangular inequalities and standard interpolation inequalities.

Remark 3.9 Since it is now known that $T$ has a fixed point $\left(u_{h}, \sigma_{h}\right)$ with $\| u_{h}-$ $I_{h} \sigma \|_{L^{\infty}} \leq C h^{k-1}$, it should be possible to derive a $\mathrm{O}\left(h^{k}\right)$ error estimate in the $H^{1}$ norm for $u-u_{h}$ by using [3, Lemma 3.10] and [3, Lemma 3.11]. Note that in the proof of [3, Lemma 3.10] an inverse estimate, used to estimate $\left\|u_{h}-I_{h} \sigma\right\|_{L^{\infty}}$ from $\left\|u_{h}-I_{h} \sigma\right\|_{L^{2}}$ can now be avoided.

Acknowledgements The author was partially supported by NSF Grant DMS-1319640. The author thanks Eric Malitz for many useful discussions about Reference [2] and Hengguang Li for discussions on the Ritz projection.

\section{References}

1. Awanou, G.: Quadratic mixed finite element approximations of the Monge-Ampère equation in 2D. Calcolo 52(4), 503-518 (2015)

2. Awanou, G.: Standard finite elements for the numerical resolution of the elliptic Monge-Ampère equations: classical solutions. IMA J. Numer. Anal. 35(3), 1150-1166 (2015)

3. Awanou, G., Li, H.: Error analysis of a mixed finite element method for the Monge-Ampère equation. Int. J. Numer. Anal. Model. 11, 745-761 (2014)

4. Babuška, I., Osborn, J., Pitkäranta, J.: Analysis of mixed methods using mesh dependent norms. Math. Comput. 35(152), 1039-1062 (1980)

5. Brenner, S.C., Scott, L.R.: The mathematical theory of finite element methods, Texts in applied mathematics, vol. 15, 2nd edn. Springer-Verlag, New York (2002)

6. Butzer, P.L., Berens, H.: Semi-groups of operators and approximation. Die Grundlehren der mathematischen Wissenschaften, Band, vol. 145. Springer-Verlag New York Inc, New York (1967)

7. Cameron, A.W.: Estimates for solutions of elliptic partial differential equations with explicit constants and aspects of the finite element method for second-order equations. Dissertation, Cornell University (2010)

8. Crouzeix, M., Thomée, V.: The stability in $L_{p}$ and $W_{p}^{1}$ of the $L_{2}$-projection onto finite element function spaces. Math. Comput. 48(178), 521-532 (1987)

9. DeVore, R., Scherer, K.: Interpolation of linear operators on Sobolev spaces. Ann. Math. (2) 109(3), 583-599 (1979)

10. Douglas Jr., J., Dupont, T., Wahlbin, L.: The stability in $L^{q}$-projection into finite element function spaces. Numer. Math 23, 193-197 (1974/75)

11. Gilbarg, D., Trudinger, N.S.: Elliptic partial differential equations of second order. Classics in Mathematics. Springer-Verlag, Berlin (2001, Reprint of the 1998 edition) 
12. Li, H.: The $W_{p}^{1}$ stability of the Ritz projection on graded meshes. Math. Comput. (2016, To appear in)

13. Neilan, M.: Quadratic finite element approximations of the Monge-Ampère equation. J. Sci. Comput. 54(1), 200-226 (2013)

14. Neilan, M.: Finite element methods for fully nonlinear second order PDEs based on a discrete Hessian with applications to the Monge-Ampère equation. J. Comput. Appl. Math. 263, 351-369 (2014)

15. Rannacher, R., Scott, R.: Some optimal error estimates for piecewise linear finite element approximations. Math. Comput. 38(158), 437-445 (1982)

16. Schatz, A.H.: Pointwise error estimates and asymptotic error expansion inequalities for the finite element method on irregular grids. I. Global estimates. Math. Comput. 67(223), 877-899 (1998)

17. Wu, Z., Yin, J., Wang, C.: Elliptic \& parabolic equations. World Scientific Publishing Co. Pte. Ltd., Hackensack (2006). doi:10.1142/6238 